\title{
Sharing Driving between a Vehicle Driver and a Sensor System using Trust-Factors to Set Control Gains
}

\author{
David Adrian Sanders TD \\ School of Engineering \\ University of Portsmouth \\ Portsmouth, UK \\ david.sanders@port.ac.uk \\ Alexander Gegov \\ School of Computing \\ University of Portsmouth \\ Portsmouth, UK \\ alexander.gegov@port.ac.uk
}

\author{
Giles Eric Tewkesbury MBE \\ School of Engineering \\ University of Portsmouth \\ Portsmouth, UK \\ giles.tewkesbury@port.ac.uk
}

\author{
Rinat Khusainov \\ School of Engineering \\ University of Portsmouth \\ Portsmouth, UK \\ rinat.khusainov@port.ac.uk
}

\begin{abstract}
A system is presented that shares control between a driver and sensors to maneuver a vehicle. The vehicle can be driven by a driver, or by the sensors, or they can share control between them. In some circumstances, sharing control can allow a human driver to drive more efficiently and safely. The gains in the controller are automatically set for the human driver and sensors by calculating a trust-factor for the vehicle driver. The sensors can assist a human driver in driving the vehicle to offset for any detected shortcomings; for example, the driver may not be able to see a vehicle ahead or the human driver may be tired. In emergencies, efficient interaction between the vehicle and a human driver can make all the differences. This research explores that collaboration and interfacing. The proposed methods are validated with initial testing.
\end{abstract}

Keywords-Vehicle; driver; trust-factor; shared-control

\section{INTRODUCTION}

Advanced driver-assistance systems automate, adapt and enhance vehicle systems to improve driving and safety. Safety features can avoid collision or accidents. Technology can be used to avoid collisions and / or alert drivers about difficulties and can take over all or some control of a vehicle. Features can include adaptive cruise control and automatic braking or can alert a driver about other cars on the road or about potential danger. They can keep a vehicle in the correct lane or consider and / or reveal objects in a blind spot. In emergencies, efficient interaction between the vehicle and a human driver can make all the difference. This research explores that collaboration and interfacing [1]-[8].

Some driver-assistance systems features are being built in to some modern cars and others can be available as optional add-on packages or as after-market solutions [9]. Driverassistance systems rely on inputs from sensors such as radar, imaging and vision, in-car networking and Light Detection and Ranging (LiDAR).
Driver-assistance is fast growing [10], [11] with increasing adoption rates and specific standards being developed. For example, IEEE 2020 image quality and communications protocols such as Vehicle Information Application Programming Interface (API) [12].

In addition, vehicle-to-infrastructure and vehicle-to-vehicle systems can be added; for example, Wi-Fi, mobile telephony or data networks [13].

Many systems have been described in the literature for helping human drivers to work in hazardous or remote environments [14]-[22]. Research has explored the way that drivers can cooperate with vehicles [23]-[25]. Controllers have tended to trade stability margin for clarity [26]-[28].

Unstructured environments can make operation more difficult for vehicles [29]-[35]. A wheeled vehicle has been studied in this paper because that remains the most common type [36]-[42].

A simple control input device for testing vehicle control has been a low current joystick. A vehicle then draws a higher current to drive motors. A human driver is usually better at driving than a computer so the systems described here attempt to assist a human driver.

Driving tends to be open-loop. A human driver indicates a desired speed and direction and their vehicle attempts to travel at that speed and on that bearing. Differences between the wheels on the vehicle or different responses to a variety of gradients and surfaces can disturb the path. Drivers need to react to the disturbances and correct the vehicle path.

Unpredictable situations can happen [43] that might affect a vehicle driver and vehicle operation [44]. Collaboration between the sensor systems on board the vehicle and a human driver [45] can help the driver [46]. 
Kuniaki [47] presented a collaborative system and Macharet [48] presented a sensor system using ultrasonics to show the bearings to targets using triangulation and those ideas were extended in [49], [50].

Methods described here allow intimate collaboration between sensors and a vehicle driver because of merging human driver commands with data from the vehicle sensors. A combined control architecture is explained in [51] and IntelliSys paper and IEEE journal that promotes cooperation between a vehicle and driver. In [52], combined control is described that improves performance and reduces workload by providing feedback from an automated process. Autonomous systems can assist a driver to improve reduce workload and improve safety for vehicles [53], [54]. Satti [55] described combined control employing computer-brain interfaces. A controller is presented in [56] that controls quadcopters and that can avoid collisions and fly the copters in formation.

A human driver and the sensors can provide commands simultaneously and in that case they can be fused using specified ratios [57]. How authority is distributed is significant for efficient co-operation. Numerous interfaces might be employed [58]-[64]

In [65], Carlson et al described methods to predict a direction of travel and change the control signals to make a vehicle move in that direction. Experimentation provided the parameters and dynamic-distribution adjusted the distribution of control in real-time. In later work the weights were changed after evaluating the commands from a human operator.

Methods to assist a vehicle driver in complex and changing environments are introduced in this paper. By combining suggestions from sensors and commands from a human driver, both the driver and the sensors cooperate to produce safe movements. The sensor system knows the vehicle status and then directs the vehicle to turn. The vehicle moves in that direction but avoids obstructions along the way.

Section II is a description of the vehicle and sensors used to test the ideas. The new controller is described in Section III and shared control in Section IV. Section V discusses the results and Section VI summarises work. Finally, some future work is suggested in Section VII.

\section{VEHICLE AND SENSORS}

This research used a Bobcat II Vehicle [22], [26] consisting of: inputs from a driver and sensors, the vehicle base, ultrasonic sensors used to avoid obstacles, and the shared controller. Ultrasonic sensors provided ranges to obstacles ahead of the vehicle. Data from the sensors were processed by a computer that adjusted the speed and direction of the vehicle base.

\section{A. Vehicle}

The vehicle base had four wheels; two large driving wheels at the front and two casters at the back. Each driving wheel was attached to a motor and could be driven independently.

The vehicle was steered by changing the current sent to each wheel motor. The vehicle could turn on its center-of- rotation. If $\mathrm{V}$ is vehicle linear velocity, $\omega$ is angular velocity and $\phi$ is direction, then velocity at the center of mass of the vehicle base is

$$
\mathrm{V}_{\mathrm{c}}=(\mathrm{V}, \phi, \omega)
$$

The kinematic model is explained in [3].

Independently driving the wheels produce orientation and movement because the driving-wheels of the vehicle were on the same axis.

\section{B. Sensor System}

Ultrasonic sensors detected obstacles ahead of the vehicle. The transmitters needed a $3 \mathrm{~m} \mathrm{~s}$ pulse to achieve the highest output. Long pulses held more energy and could detect obstacles at longer ranges. If the speed of sound is assumed to be $330 \mathrm{~m} / \mathrm{s} \ldots$ then a $3 \mathrm{~ms}$ sound pulse is $0.99 \mathrm{~m}$ long. Permitting a pulse to exit from its transmitter, rebound back from an obstacle and reappear back at a receiver, suggests $0.5 \mathrm{~m}$ is a minimum range for a $3 \mathrm{~ms}$ pulse. The work needed ranges that were closer and so various shorter pulse lengths were used.

Obstacles appeared and disappeared when the vehicle travelled about and it was sometimes challenging to lock on to a target. Ultrasonics were noisy and returned some misreads. Misreads were filtered out to improve reliability. Histogramic In-Motion Mapping was used. Volumes ahead of the vehicle were separated in to a 3 sector grid and stored in an array: far, middle and near. Ultrasonic transducers were fixed so that they overlapped and covered the area ahead. Array elements were incremented by five if they contained an obstacle. Array elements that did not contain an obstacle were decremented by 1. Fig. 1 illustrates beam patterns for two ultrasonic sensors.

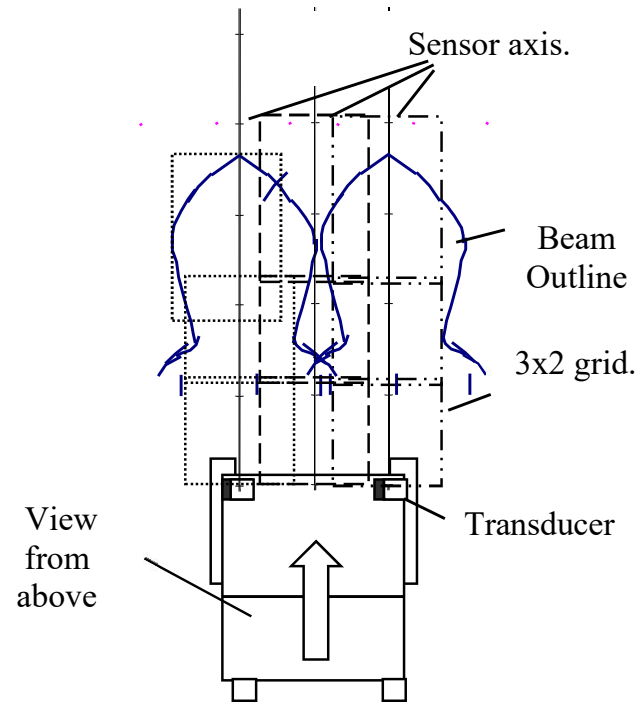

Fig. 1. Overlapping beams from two ultrasonic sensors to create an array.

Arrays had a minimum quantity of 0 and maximum of 15 . Fig. 2 symbolizes a 3-element histogrammic depiction of a local environment. An obstacle in the $3^{\text {rd }}$ element is making it increase. 


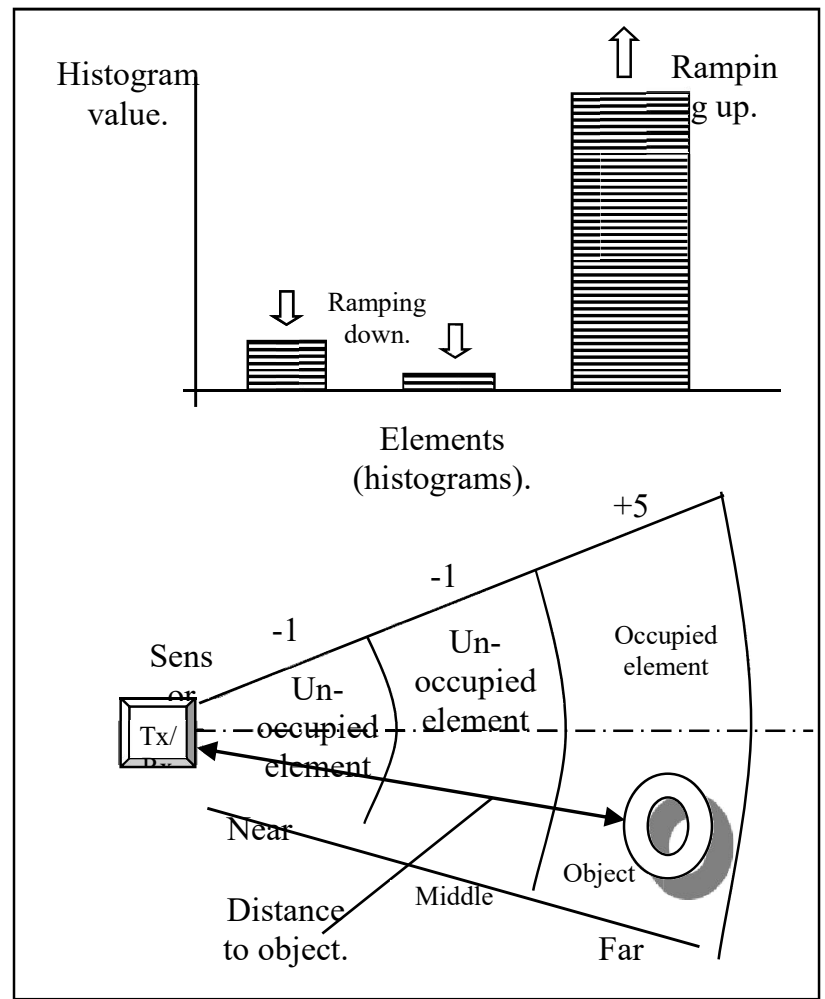

Fig. 2. 3-element histogrammic depiction of a local environment. An obstacle in the 3rd element is making it increase.

An obstacle within a grid element caused the element to rapidly increase in value to the highest value. Arbitrary misreads within the other elements increased values briefly, but they were decremented during every system update period. If the obstacle relocated to another element, then that element rapidly increased in value and the previous one reduced in value. Reliable ranges were acquired within $0.5 \mathrm{~s}$.

\section{CONTROL}

A controller drove the vehicle following commands from a driver and the sensor system automatically avoided obstacles in the vehicle path.

\section{A. Controller}

Angular velocity and linear velocity of the vehicle were considered. The vehicle followed a desired direction and linear velocity when the vehicle was at an arbitrary heading angle, as shown in Fig. 3.

The control law to track the target position for the vehicle's linear velocity, $V_{\mathrm{r}}$ was:

$$
\begin{aligned}
& V_{\mathrm{r}}=\mathrm{V}_{\mathrm{M}} \times \mathrm{D} / \mathrm{D}_{\text {Des }} \text { when }|\mathrm{D}|<\mathrm{D}_{\text {Des }} \\
& \text { and } \\
& V_{\mathrm{r}}=\mathrm{V}_{\mathrm{M}} \times \mathrm{D} / \mathrm{D}_{\text {DesSp }} \text { when }|\mathrm{D}|>\mathrm{D}_{\text {DesSp }}
\end{aligned}
$$

Where, $V_{M}$ is the maximum speed of the vehicle, $\mathrm{D}$ is a vector from the vehicle joystick, and $\mathrm{D}_{\text {Des }}$ is the demanded speed. If the vector from the vehicle joystick is greater than the sensor range, the vehicle moves at the desired speed.

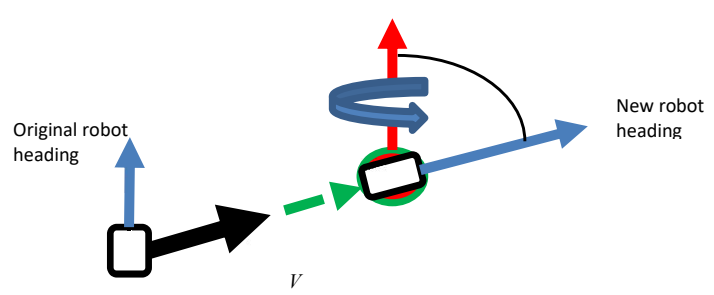

Fig. 3. Controller design.

The vehicle's heading changes during traveling. To track the vehicle's heading, the control law for the vehicle's angular velocity, $\omega_{r}$, is defined as:

$$
\omega_{r}=\omega_{\mathrm{M}} \times \Delta \theta / \theta_{\mathrm{D}} \text {, when }|\Delta \theta|<\theta_{\mathrm{D}}
$$

and

$$
\omega_{r}=\omega_{\mathrm{M}} \mathrm{x} \Delta \theta /|\Delta \theta|, \quad \text { when }|\Delta \theta|>\theta_{\mathrm{D}}
$$

Where, $\theta_{\mathrm{D}}$ is the desired heading, $\omega_{\mathrm{M}}$ is the maximum value of the vehicle's angular velocity and the direction of the vehicle is expressed as a vehicle heading error, $\Delta \theta$.

If the vehicle's heading error is greater than a buffer angle, the vehicle turns. If the vehicle's heading error is less than a buffer angle, the control law adjusts angular velocity to track the desired heading.

\section{B. Avoiding Obstacles}

The omnidirectional mobility of the vehicle made obstacle avoidance easier. A vector represented speed and direction. A repulsive force was generated if the vehicle drove near to an obstacle and the vehicle steered away from the object (Fig. 4).

The avoidance velocity, $\mathbf{V}_{\mathrm{o}}$ was:

$$
\mathbf{V}_{\mathrm{O}}=a \sum_{\mathrm{I}}\left[\left(\mathrm{D}_{\mathrm{Ms}^{-}}\left|\boldsymbol{x}_{\mathrm{i}}\right|\right) / \mathrm{D}_{\mathrm{s})}\left(-\boldsymbol{x}_{\mathrm{i}} /\left|\boldsymbol{x}_{\mathrm{i}}\right|\right]\right.
$$

Where, $\mathrm{D}_{\mathrm{s}}$ was a safe distance, $\boldsymbol{x}_{\mathrm{i}}$ were vectors to represent objects ahead of the vehicle, and $a$ was a constant. Ultrasonic sensors detected the positions of obstacles, $x_{i}$.

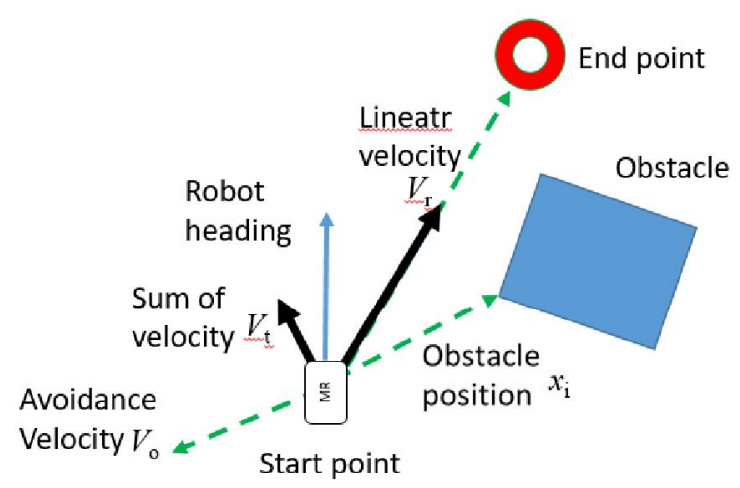

Fig. 4. A repulsive force was generated if the vehicle drove near to an obstacle and the vehicle steered away from the object. 
The resultant obstacle velocity, $\boldsymbol{V} t$, was:

$$
\boldsymbol{V}_{t}=+\boldsymbol{V}_{o}
$$

Where, $\boldsymbol{V}_{o}$ was an avoidance velocity that the system generated, $\boldsymbol{V}_{r}$ was linear velocity produced by destination seeking, and, $\boldsymbol{V}_{t}$ was the resultant velocity. $\boldsymbol{V}_{t}$ avoided obstacles but did not change the heading of the vehicle much.

\section{SHARED CONTROLLER}

Shared-control combined sensor system commands and commands from the driver to improve driving.

A driver could generally control a vehicle safely but the sensors were more accurate and repeatable. The systems gave autonomy to the human driver and used their skills when possible but intervened if necessary to avoid obstacles.

When the vehicle operated in varying and complex environments, then the system provided better decisionmaking. The shared and combined-control architecture is shown in Fig. 5.

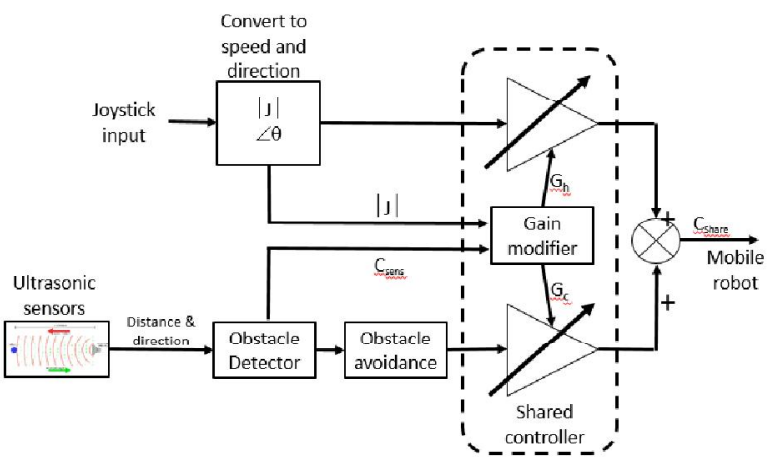

Fig. 5. Shared and combined control

The shared and combined control extends work described in [53]. It allowed convenient and safe maneuvering of a vehicle.

The architecture combined a joystick input and shared it with sensor inputs. The driver controlled the vehicle using a joystick and could usually see the vehicle. The vehicle sensor system avoided obstacles and ensured safety when the vehicle moved.

Current to the vehicle motors was generated by both a driver and sensors. When obstacles were far away, a human driver did not need assistance. In environments with many objects or objects near to the vehicle, the system reduced or inhibited commands from the joystick given by the driver so as to avoid collisions.

The combined-control gains from a driver and sensors changed as the vehicle moved around. The resultant control command, $\boldsymbol{C}_{\text {share }}$ (Fig. 5) was:

$$
C_{\text {share }}=G_{h}|\mathrm{~J}|+G_{c}, C_{\text {sens }} \in[0,1]
$$

Where, $C_{\text {sens }}$ was a range to an object and $|\jmath|$ was the input from the joystick. $\boldsymbol{C}_{\text {share, }}$ was added to the weighted driver joystick input multiplied by a weighted gain $G_{h}$, and a weighted output from the autonomous controller, $G_{\mathrm{w}}$ was the weighted gain. Confidence-factors established the gains. The system considered the Trust-factor of the driver to determine a driver gain as in (13).

\section{A. Avoidance Confidence}

Confidence of the driver was estimated. The Trust-factor was made up of three Factors. An Avoidance Factor was set to represent the ability of a driver. Drivers were given a lower Trust-factor when the vehicle moved closer to an object. $E_{a v}$, the avoidance-factor, was

$$
E_{a \mathrm{v}}=\left|x_{0}\right| / \mathrm{D}_{\mathrm{Sa}}
$$

Where, $\mathrm{D}_{\mathrm{Sa}}$ was a constant representing a cautious and safe range and $x_{o}$ is the shortest distance between an object and the vehicle. If the powered vehicle was further away from an object than $\mathrm{D}_{\mathrm{Sa}}$, then the driver was given a greater confidence rating in driving the vehicle. If a vehicle was at a distance less than $\mathrm{D}_{\mathrm{Sa}}$ from an object, then confidence decreased.

\section{B. Safety Confidence}

The Safety Factor denoted the ability of the driver to safely drive a vehicle. If the vehicle was operating at low speed, the driver was assumed to be more confident. A Trust-factor for safety $E_{\text {safe was: }}$

$$
E_{\text {safe }}=\left\{1-\left(\left|V_{\mathrm{h}}\right| /\left|V_{\mathrm{TH}}\right|\right)\right\}^{q} \text {, for } q<1 .
$$

Where, threshold $V_{T H}$ is the fastest linear velocity that a user was permitted to drive a vehicle and $V_{h}$ was the user's command linear velocity from their joystick. To assign a greater Trust-factor at low speed, it is projected by means of an exponent, $\mathrm{q}$ (where $\mathrm{q}<1$ ).

\section{Assistance Confidence}

Tiredness and time were important. If a human driver controlled a vehicle continuously, the driver was liable to grow tired. In that case, their Trust-factor reduces. A driver was more likely to be alert and awake at the start of a day. Joystick control for an entire day was monitored. Engagement time, $E_{\text {te, }}$, is

$$
\begin{aligned}
& E_{t \mathrm{e}}=E_{t \mathrm{e}-1}+\left(1 / T_{\mathrm{a}}\right), \text { if driver is rested } \\
& E_{t \mathrm{e}}=E_{t \mathrm{e}-1}-\left(1 / T_{\mathrm{a}}\right), \text { if driver is tired }
\end{aligned}
$$

Where, $T_{\mathrm{a}}$ is the time that a driver has been driving a vehicle. If a driver actively controlled a vehicle then estimation slowly dropped. If a driver rested, then estimation increased.

\section{Overall Confidence}

Control gains $\mathrm{G}_{c}$ and $\mathrm{G}_{h}$ and the overall Trust-factor were:

OverConFact $=E_{t} \times \max \left(E_{\text {avoid, }}, E_{\text {safety }}\right)$

$$
\mathrm{G}_{h}=\text { OverConFact }
$$

$\mathrm{G}_{c}=1-$ OverConFact

When both safety and avoidance trust-factors were high, a driver would drive their vehicle smoothly. When avoidance was high, the vehicle was far away from objects in its path and 
the driver had complete control of their vehicle. When safety estimates were higher, the speed of the vehicle was limited.

\section{RESULTS}

Experiments were undertaken to validate the methods.

\section{A. Simulation}

Simulation validated the vehicle shared-control. The input from the joystick was fixed to steer to a target destination. Speed was set to $1 / 2$ speed.

An example of a trajectory is represented in Fig. 6. Fig. 6 shows the vehicle's heading and position at numerous instants in time. At the start position, the simulated vehicle was facing right. The direction to a target destination is represented by a dotted line that is the input from the joystick. A sensor on the left of the vehicle detected a wall and guides the vehicle away. The additional solid line indicates the direction of motion; towards Point A from the Start. As the vehicle moves towards Point A, the ultrasonic sensor on the right of the vehicle detects a wall below the vehicle and turns the vehicle left to avoid it. Once in free space Vo, reduces to zero and the vehicle turns and moves towards the target destination, guided by the joystick. At Point $\mathrm{C}$ the sensor on the left of the vehicle detects a gap and $\mathrm{VoC}$ grows so that the vehicle steers right to avoid the gap edge.

At Point D and through the gap, the vehicle is safe. No obstacles are detected ahead of the vehicle. The vehicle can turn and move towards the target.

The simulated vehicle did not collide with any obstacles and safely approached the target destination.

Values for the Trust-factors were:

$\begin{array}{ll}\text { Start } & 0.4 \\ \text { A } & 0.6 \\ \text { B } & 1.0 \\ \text { C } & 0.7 \\ \text { D } & 1.0\end{array}$

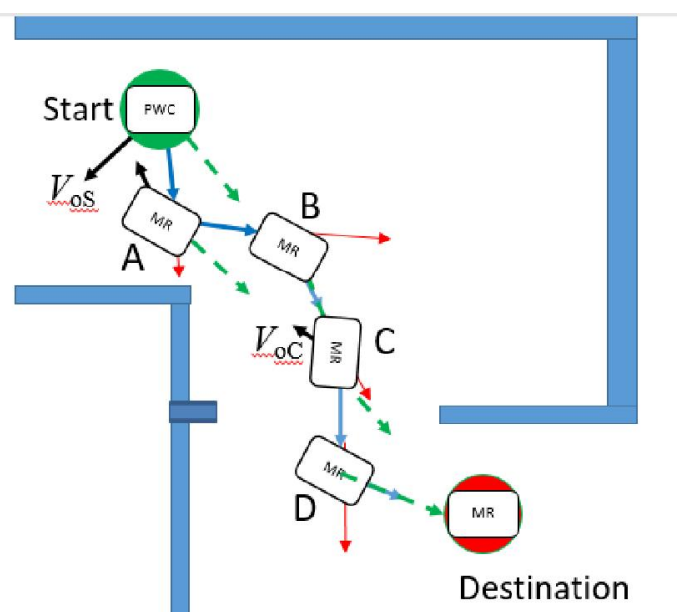

Fig. 6. Simulation experimentation and testing.
The Confidence value for avoiding obstacles was significant when objects were detected, so driver confidence was decreased when objects were detected. The vehicle reduced speed. The sensors partially controlled the vehicle until it was in open space and safe. The shared and combined control meant that the vehicle obeyed joystick commands to move towards a destination while avoiding obstacles.

The commands to the vehicle motors were an amalgamation of a simulated input from a sensor system and simulated joystick input (13). Controller gains for the sensor system and driver produced speed and steering commands for the simulated vehicle. The simulated vehicle did not crash.

\section{B. Experimenting with a Vehicle}

Volunteer drivers at Portsmouth maneuvered the vehicle past obstacles to drive to a target destination. Drivers controlled the vehicles using joysticks.

Trajectories were recorded using a camera and a typical vehicle and Fig. 7 shows a typical path. The vehicle could easily turn and was able to spin on its axis if required, before driving in a selected direction to maintain a desired heading. That allowed drivers to concentrate on steering. They did not have to concentrate on avoiding obstacles.

The ultrasonic sensors helped drivers to control their vehicles using shared and combined control. The vehicle did not collide and safely reached the target destination.

Recorded values of Trust-factor for the experiment were:

Start $\quad 0.0$

$\begin{array}{ll}\text { A } & 0.07 \\ \text { B } & 0.69 \\ \text { C } & 0.69 \\ \text { D } & 0.71\end{array}$

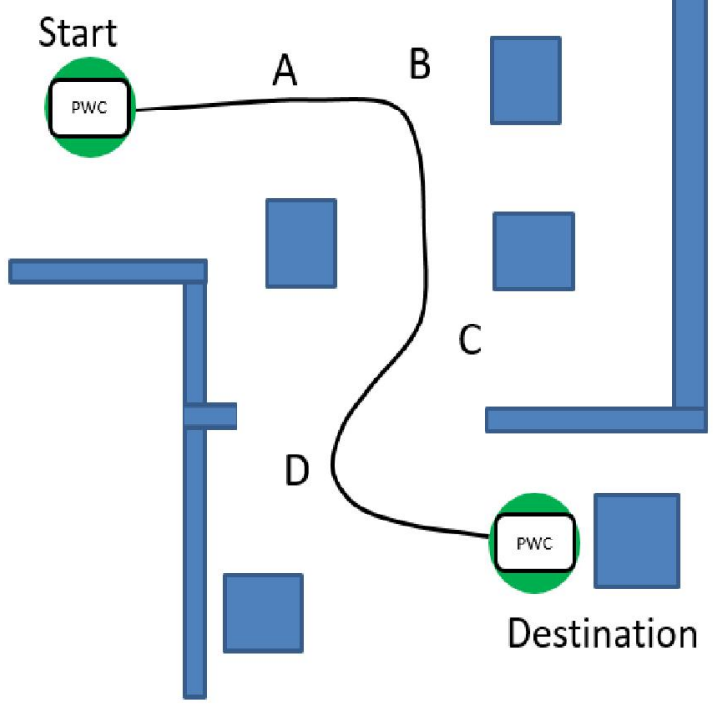

Fig. 7. Recorded trajectory of vehicle experiment. 
Control of the vehicle was a combination of inputs from the driver's joystick and from the sensors (13). Control gains for both the driver and the sensors dictated the speed of the two motors and therefor the direction that the vehicle moved in.

Driver Confidence reduced when obstacles needed to be avoided and the vehicle did not collide with anything.

If a driver slowed the vehicle then it became safer and so that driver had a higher authority. Shared-control allowed the vehicle to move away from objects while following instructions from a driver's joystick.

\section{DISCUSSION AND CONCLUSIONS}

Shared-control was implemented on a vehicle. A driver was in control of the vehicle unless sensors needed to assist, for example, to avoid an object.

The work did not deal with objects above or below the volume that could be detected by the ultrasonics in the way that they were set up on the vehicle.

The mix of the input from sensors and driver were calculated using Trust-factors. They were established using evidence from the sensors such as: range from the vehicle to an object; how long the vehicle operator has been driving, etc.

Human drivers controlled the vehicle more safely when assisted by the sensors. Experimental results showed that the shared-control method was safe.

An optimal mix of human versus autonomous control exists for different vehicle drivers in various conditions, for example, whether a driver is tired. The most favorable mix changed with human experience and skill.

\section{FUTURE WORK}

The static ultrasonic sensor array is limiting ongoing work and a scanning device has been created at Chailey Heritage (by Martin Langner). Future research will use that device as it is smaller, covers a bigger volume and range and position can be detected more accurately. Different AI methods are being investigated but they are tending to be more complicated.

\section{REFERENCES}

[1] D.A. Sanders, Ndzi, D, Chester, S \& Malik, M 2017, Adjustment of teleoperator learning when provided with different levels of sensor support while driving mobile robots. in $\mathrm{Y}$ Bi, S Kapoor \& $\mathrm{R}$ Bhatia (eds), SAI Intelligent Systems (IntelliSys) vol. 2, Lecture Notes in Networks and Systems, vol. 16, Springer, UK, pp. 548-558. DOI: 10.1007/978-3-31956991-8_41. 2017

[2] D.A. Sanders, Sanders, H, Gegov, A \& Ndzi, D 2017, Rule-based system to assist a tele-operator with driving a mobile robot. in $\mathrm{Y} \mathrm{Bi}, \mathrm{S}$ Kapoor \& R Bhatia (eds), SAI Intelligent Systems (IntelliSys) vol. 2, Lecture Notes in Networks and Systems, vol. 16, Springer, pp. 599-615, UK. DOI: 10.1007\%2F978-3-319-56991-8 44. 2017.

[3] D.A. Sanders, 'Using self-reliance factors to decide how to share control between human powered wheelchair drivers and ultrasonic sensors' IEEE Transactions on Neural Systems and Rehabilitation Engineering, vol 25, no. 8, pp. 1221-1229. 2017.

[4] D. A. Sanders, "Non-model-based control of a wheeled vehicle pulling two trailers to provide early powered mobility and driving experiences", IEEE Transactions on Neural Systems and Rehabilitation Engineering, vol 26, no. 1, pp. 96 - 104. 2018.

[5] D.A. Sanders, Gegov, A, Tewkesbury, G \& Khusainov, R, Rule-based system to assist a powered wheelchair driver. IEEE Proceedings of the
Intelligent Systems Conference (IntelliSys) 2017. IEEE, London, United Kingdom, 7-8 September. 2017.

[6] D.A. Sanders, Sanders, B, Gegov, A \& Ndzi, D, Results from investigating powered wheelchair users learning to drive with varying levels of sensor support. in Proceedings of the Intelligent Systems Conference (IntelliSys) 2017. IEEE, London, UK, 7-8 September. 2017.

[7] D.A. Sanders, Sanders, B, Ndzi, D \& Bausch, N, Using confidence factors to share control between a mobile robot tele-operater and ultrasonic sensors. in Proceedings of SAI Intelligent Systems Conference (IntelliSys) 2017. IEEE, London, UK, 7-8 September. 2017,

[8] D.A. Sanders, Gegov, A \& Ndzi, D, Knowledge-based expert system using a set of rules to assist a tele-operated mobile robot. in $\mathrm{Y} \mathrm{Bi}, \mathrm{S}$ Kapoor \& R Bhatia (eds), Intelligent Systems and Applications. Studies in Computational Intelligence, vol. 751, Springer. 2017.

[9] US: Mobileye intros smartphone connected driver assistance (ADAS) technology". Telematics News 2012

http://telematicsnews.info/2012/01/12/us-mobileye-intros-smartphoneconnected-driver-assistance-adas-technology_j3122. Retrieved $13 / 11 / 2017$.

[10] I. Riches. "Strategy Analytics: Automotive Ethernet: Market Growth Outlook | Keynote Speech 2014 IEEE SA: Ethernet \& IP @ Automotive Technology Day" (PDF). IEEE.

http://standards.ieee.org/events/automotive/2014/00 Automotive Ethern et Market_Growth Outlook.pdf. Retrieved 13/11/2017.

[11] K. Rees and J S Park, Vehicle Information Access API". W3C Business Group report, W3C and LG Electronics. 2014 https://www.w3.org/2014/automotive/vehicle_spec.html. Retrieved $13 / 11 / 2017$.

[12] ADAS Definition". Autoconnectedcar.com. Archived from the original on $06 / 10 / 2012$. https://web.archive.org/web/20120610055853/http://telematicsnews.info /2012/01/12/us-mobileye-intros-smartphone-connected-driverassistance-adas-technology_j3122. Retrieved 13/11/2017.

[13] Banga, Bhavya, Global ADAS and Autonomous Driving Components Market, Analysis \& Forecast. Business Intelligence and Strategy Research. 2017. https://bisresearch.com/industry-report/global-adasautonomous-driving-components-market-2026.html. Retrieved $13 / 11 / 2017$

[14] D.A. Sanders, The modification of pre-planned manipulator paths to improve the gross motions associated with the pick and place task, ROBOTICA, vol. 13 pp.77-85. 1995.

[15] T.B. Sheridan, Tele-operation, telerobotics and telepresence: A progress report, Control Eng. Pract. 3(2), pp 205-214. 1995.

[16] T. Sheridan, Humans and Automation: System Design and Research Issues. John Wiley and Sons. 1995.

[17] D.A. Sanders, Comparing speed to complete progressively more difficult mobile robot paths between human tele-operators and humans with sensor-systems to assist. ASSEMBLY AUTOM, vol. 29, no. 3, pp. 230-248. 2009.

[18] D.A. Sanders, M. Langner M and G.E. Tewkesbury, Improving wheelchair-driving using a sensor system to control wheelchair-veer and variable-switches as an alternative to digital-switches or joysticks. IND ROBOT, vol. 37, no. 2, pp. 157-167, 2010.

[19] D.A. Sanders and A. Baldwin, X-by-wire technology, Total Vehicle Technology Conference, pp. 3-12. 2001.

[20] D.A. Sanders, Controlling the direction of walkie type forklifts and pallet jacks on sloping ground. ASSEMBLY AUTOM, vol. 28 (4), pp 317-324. 2008.

[21] P. Fiorini and R. Oboe, Internet-based telerobotics: Problems and approaches, Proc' of ICAR'97, Monterey, CA, pp. 765-770. 1997.

[22] J.P. Richard, Time-delay systems: An overview of some recent advances and open problems, Automatica vol. 39, pp 1667-1694. 2003.

[23] D.A Lawrence, Stability and transparency in bilateral tele-operation, IEEE Trans. Robot. Autom. Vol. 9 (5). 1993.

[24] W. Kim, B. Hannaford and A. Bejczy, Force reflection and shared compliant control in operating telemanipulators with time delay IEEE Trans. Robot. Autom. vol. 8(2), 176-185. 1992.

[25] G. Niemeyer and J.J Slotine, Stable adaptive tele-operation. IEEE J. 
Ocean. Eng. vol. 16(1), 152-162. 1991

[26] G. Niemeyer and J.J Slotine, Towards force reflecting tele-operation over the Internet, Proc' of the IEEE Int' Conf' on Robotics and Automation, Leuven, Belgium (ICRA'98), pp. 1909-1915. 1998.

[27] B.L. Luk, D.S. Cooke, S. Galt, A.A Collie and S. Chen, Intelligent legged climbing service robot for remote maintenance applications in hazardous environments, Jrnl of Robotics and Autonomous Systems, vol. 53/2, pp. 142-52. 2005.

[28] B. Rooks, Plotting future UK robotics research Programmes, IND ROBOT vol. 33 (3), pp. 165-169. 2006.

[29] D.A. Sanders, GE Tewkesbury and DC Robinson, Simple expert systems to improve an ultrasonic sensor-system for a tele-operated mobile-robot. SENSOR REV, vol. 31, no. 3, pp. 246-260. 2011.

[30] D.A Sanders and I.J. STOTT, Analysis of failure rates with a teleoperated mobile robot between a human tele-operator and a human with a sensor system to assist. Robotica Journal. 30 (6) 2012 , pp. 973-988.

[31] D Sanders, G. Tewkesbury and J. Graham-Jones,Simple rules to modify pre-planned paths to improve gross robot motions associated with pick \& place assembly tasks. ASSEMBLY AUTOM, vol. 31 (1) pp. 69-78 2011.

[32] D.A. Sanders, Comparing ability to complete simple tele-operated rescue or maintenance mobile-robot tasks with and without a sensor system. SENSOR REV, vol. 30, no. 1, pp. 40-50. 2010.

[33] D.A. Sanders, J. Graham-Jones and A. Gegov, Improving ability of teleoperators to complete progressively more difficult mobile robot paths using simple expert systems and ultrasonic sensors. IND ROBOT, vol. 37, no. 5, pp. 431-440. 2010.

[34] I. Stott and D. Sanders, New powered wheelchair systems for the rehabilitation of some severely disabled users, Int Jrnl REHAB RESEARCH vol. 23 (3), pp. 149-153. 2000.

[35] D. Sanders and I. Stott, A new prototype intelligent mobility system to assist powered wheelchair users IND ROBOT vol. 26 (6), pp. 466-475. 1999.

[36] M.J Goodwin, D.A. Sanders and G.A. Poland et al, Navigational assistance for disabled wheelchair-users, proc' of Euromicro Conference 95 vol. 43, pp. 73-79. 1997.

[37] D. Sanders, Analysis of the effects of time delays on the teleoperation of a mobile robot in various modes of operation, IND ROBOT vol. 36 (6) pp. 570-584. 2009.

[38] D. Sanders, I. Stott, J Graham-Jones et al, Expert system to interpret hand tremor and provide joystick position signals for powered wheelchairs with ultrasonic sensor systems, IND ROBOT vol. 38 (6), pp. 585-598. 2011.

[39] D.A. Sanders, N. Bausch, H. Liu, et al, Improving Steering of a Powered Wheelchair Using an Expert System to Interpret Hand Tremor, Proc' Intelligent Robotics \& Applications, Pt I vol. 9245 pp. 460-471. 2015.

[40] D. Sanders and I. Stott, The use of virtual reality to train powered wheelchair users and test new wheelchair systems, International Journal of Rehabilitation Research vol. 23 (4), pp. 321-326. 2000.

[41] D. Sands, Cost effective robotics in the nuclear industry. IND ROBOT, vol. 33, No 3, pp 170 - 173. 2006.

[42] T. Nakamura and K. Satoh, Development of an omni-directional mobile robot using traveling waves based on snail locomotion. IND ROBOT, vol. 35 (3), pp. 206-210. 2008.

[43] B.L Luk, A.A. Collie, D.S Cooke and S.Chen Walking and climbing service robots for safety inspection of nuclear reactor pressure vessels. Measurement \& Control vol. 39 (2), pp. 43-47. 2006.

[44] B. Luk, K. Liu, A. Collie, D. Cooke \& S. Chen, Tele-operated climbing and mobile service robots for remote inspection and maintenance in nuclear industry, IND ROBOT, vol. 33, No 3, pp. 194 - 204. 2006.

[45] M.J Bakari and D.W Seward, Human arm-like mechanical manipulator the design and development of a multi-arm mobile robot for nuclear decommissioning. Proc' 3rd Int, Conf Informatics in control, automation and robotics - Robotics and Automation, pp. 168-175. 2006.

[46] B.P DeJong E.L Faulring, J Edward Colgate, M.A Peshkin, H. Kang, Y.S Park and T.F Ewing, Lessons learned from a novel tele-operation test-bed. IND ROBOT, vol. 33, No 3, pp. 187 - 193. 2006.
[47] D. A. Sanders, I. J. Stott, D. C. Robinson, et al., "Analysis of successes and failures with a tele-operated mobile robot in various modes of operation", Robotica, vol. 30, pp. 973-988, 2012.

[48] I. J. Stott and D. A. Sanders, "New powered mobile robot systems for the rehabilitation of some severely disabled users", Int Jrnl of Rehab Research, vol. 23, no. 3, pp. 149-153, 2000.

[49] D. A. Sanders, J. Graham-Jones, and A. Gegov, "Improving ability of tele-operators to complete progressively more difficult mobile robot paths using simple expert systems and ultrasonic sensors", Industrial Robot-an International Journal, vol. 37, no. 5, pp. 431-440, 2010.

[50] D. A. Sanders and I. J. Stott, "A new prototype intelligent mobility system to assist powered mobile robot users", Industrial Robot, vol. 26, no. 6, pp. 466-475, 1999.

[51] S. Hayati and S. T. Venkataraman, "Design and Implementation of a Robot Control System with Traded and Shared Control Capability," in Proc. of IEEE International Conf on Robotics and Automation, Scottsdal , AZ, USA, 1989, pp. 1310-1315.

[52] A. David M. M. Abbink, and E. R. Boer, "Haptic Shared Control: Smoothly Shifting Control Authority?," Cognition, Technology \& Work, Springer-Verlag, vol. 14, no. 1, pp. 19-28, 2012.

[53] Makoto Itoh, Toshiyuki Inagakia and Hiroto Tanaka, "Haptic Steering Direction Guidance for Pedestrian-Vehicle Collision Avoidance," in Proc. of IEEE Int Conf on System, Man, and Cybernetics, Seoul, Korea, 2012, pp. 3309-3314.

[54] T. Carlson and Y. Demiris, "Increasing Robotic Mobile robot Safety With Collaborative Control: Evidence from Secondary Task Experiments," in Proc. of IEEE International Conf on Robotics and Automation, Anchorage, Alaska, USA, 2010, pp. 5582-5587.

[55] A. R. Satti, D. Coyle and G. Prasad, "Self-paced Brain-controlled Mobile Robot Methodology with Shared and Automated Assistive Control," in Proc. of IEEE Symposium on Computational Intelligence, Cognitive Algorithms, Mind, and Brain, Paris, France, 2011, pp. 1-8.

[56] A. Franchi, C. Secchi, M. Ryll, H. H. Bulthoff and P. R.Giordano, "Shared Control : Balancing Autonomy and Human Assistance with a Group of Quadrotor UAVs, "IEEE Robotics \& Automation Magazine, vol.19, no.3, pp. 57-68, 2012.

[57] H. K. Kim, S. J. Biggs, D. W. Schloerb, J. M. Carmena, M. A. Lebedev, M. A. L. Nicolelis and M. A. Srinivasan, "Continuous Shared Control for Stabilizing Reaching and Grasping With Brain-Machine Interfaces," IEEE Transactions on Biomedical Engineering, vol. 53, no. 6, pp. 1164-1173. 2006.

[58] D.A. Sanders, S.D. Urwin-Wright, G.E. Tewkesbury, GE; et al. "Pointer device for thin-film transistor and cathode ray tube computer screens", Electronics Letters, vol. 41, no. 16, 2005, pp. 894-896.

[59] D.A. Sanders, "Analysis of the effects of time delays on the teleoperation of a mobile robot in various modes of operation", Ind Robot vol. 36, no. 6, 2009, pp. 570-584.

[60] D.A. Sanders, "Controlling the direction of "walkie" type forklifts and pallet jacks on sloping ground", Assembly Automation, vol. 28, no. 4, 2008, pp. 317-324.

[61] D.A. Sanders and J Bergasa-Suso, "Inferring Learning Style from the Way Students Interact with a Computer User Interface and the WWW", IEEE Transactions on Education vol. 53, no. 4, 2010, pp. 613-620.

[62] D.A. Sanders and G.E Tewkesbury, "A pointer device for TFT display screens that determines position by detecting colours on the display using a colour sensor and an Artificial Neural Network", Displays, vol. 30, no. 2, 2009, pp. 84-96.

[63] D.A. Sanders and A Baldwin, "X-by-wire technology", Total Vehicle Technology Conference, ISBN:1-86058-324-5, pp. 3-12. 2001.

[64] D.A. Sanders, "Comparing ability to complete simple tele-operated rescue or maintenance mobile-robot tasks with and without a sensor system", Sensor Review vol. 30, no. 1, 2010, pp. 40-50.

[65] T. Carlson and Y. Demiris, "Collaborative Control for a Robotic Mobile robot: Evaluation of Performance, Attention, and Workload," IEEE Trans. on Systems, Man, and Cybernetics-Part B: Cybernetics, vol. 42, no. 3, 2012, pp. 876-888. 